\title{
Prevenção de morte súbita em lactentes: uma revisão bibliográfica
}

\author{
Prevention of sudden infant death: a bibliographic review
}

Prevención de la muerte súbita del lactante: revisión bibliográfica

Priscila Libman ${ }^{1 *}$, Ana Julia Fernandes Calzolari², Bárbara Luiza Silva Alves ${ }^{3}$, Camila Quiza Vargas ${ }^{1}$, Emílio Pandeló Lima ${ }^{4}$, Erick Del'Amanche Avelino Ribeiro e Silva ${ }^{5}$, Igor Faria Reis ${ }^{6}$, Mayara dos Santos Leandro ${ }^{7}$, William Faustino da Conceição ${ }^{4}$, Diogo Pena Moreira ${ }^{8}$.

\section{RESUMO}

Objetivo: Ampliar o conhecimento sobre a Síndrome da Morte Súbita do Lactente (SMSL) e seus fatores de risco, com a finalidade de potencializar as medidas de prevenção contra a SMSL e consequente redução da mortalidade. Revisão bibliográfica: Essa síndrome apresenta-se como principal causa de óbito em países desenvolvidos, contudo, no Brasil, há escassez de dados estatísticos que possibilitam o estudo aprofundado. O baixo nível socioeconômico e educacional está ligado à carência de informação, algo que corrobora com o desenvolvimento dessa síndrome e reforça a necessidade das orientações aos pais e cuidadores. Fatores de risco como objetos no berço, cama compartilhada com os pais e tabagismo parental são evitáveis e diminuem consideravelmente o risco da SMSL. Causas como agentes infecciosos e malformações genéticas, principalmente cardiovasculares e cerebrais, também estão relacionadas ao surgimento da síndrome. Considerações finais: Conclui-se que o reconhecimento dos fatores de risco é indispensável para a prevenção contra a SMSL, fato que comprova a importância do papel do médico na educação dos responsáveis dos lactantes. É primordial, no entanto, destacar a limitação de estudos em epidemiologia dessa síndrome no Brasil.

Palavras-chave: Morte súbita, Lactente, Fatores de risco.

\begin{abstract}
Objective: To increase knowledge about Sudden Infant Death Syndrome (SIDS) and its risk factors, in order to enhance preventive measures against SIDS and the consequent reduction in mortality. Bibliographic review: This syndrome presents itself as the main cause of death in developed countries, however, in Brazil there is a lack of statistical data that allow for in-depth study. The low socioeconomic and educational level is linked to the lack of information, something that corroborates the development of this syndrome and reinforces the need for guidance to parents and caregivers. Risk factors such as objects in the crib, bed shared with parents and parental smoking are preventable and considerably decrease the risk of SIDS. Causes such as infectious agents and genetic malformations, especially cardiovascular and cerebral, are also related to the emergence of the syndrome. Final considerations: It is concluded that the recognition of risk factors is essential for the prevention of SIDS, a fact that proves the importance of the physician's role in the education of those responsible for nursing mothers. It is essential, however, to highlight the limitation of studies on the epidemiology of this syndrome in Brazil.
\end{abstract}

Keywords: Sudden death, Infant, Risk factors.

\footnotetext{
${ }^{1}$ Faculdade de Medicina Souza Marques (FTESM), Rio de Janeiro - RJ. *E-mail: priscilalibman@gmail.com

2 Pontifícia Católica de Minas Gerais (PUC Minas), Betim - MG.

${ }_{3}^{3}$ Universidade Federal de Juiz de Fora (UFJF), Governador Valadares - MG

4 Universidade de Vassouras (UV), Vassouras - RJ.

${ }^{5}$ Universidade Federal de Viçosa (UFV), Viçosa - MG.

${ }^{6}$ Universidade Vila Velha (UVV), Vila Velha - ES.

7 Universidade de Ribeirão Preto (UNAERP), Guarujá - SP.

${ }^{8}$ Centro Universitário de Caratinga (UNEC), Caratinga - MG.
} 


\section{RESUMEN}

Objetivo: Incrementar el conocimiento sobre el Síndrome de Muerte Súbita del Lactante (SMSL) y sus factores de riesgo, con el fin de potenciar las medidas preventivas contra el SMSL y la consecuente reducción de la mortalidad. Revisión bibliográfica: Este síndrome se presenta como la principal causa de muerte en los países desarrollados, sin embargo, en Brasil hay una falta de datos estadísticos que permitan un estudio en profundidad. El bajo nivel socioeconómico y educativo está vinculado a la falta de información, algo que corrobora el desarrollo de este síndrome y refuerza la necesidad de orientación a los padres y cuidadores. Los factores de riesgo como los objetos en la cuna, la cama compartida con los padres y el tabaquismo de los padres son prevenibles y disminuyen considerablemente el riesgo de SMSL. Causas como agentes infecciosos y malformaciones genéticas, especialmente cardiovasculares y cerebrales, también están relacionadas con la aparición del síndrome. Consideraciones finales: Se concluye que el reconocimiento de los factores de riesgo es fundamental para la prevención del SMSL, hecho que demuestra la importancia del papel del médico en la educación de los responsables de las madres lactantes. Sin embargo, es fundamental resaltar la limitación de los estudios sobre la epidemiología de este síndrome en Brasil.

Palabras clave: Muerte súbita, Lactante, Factores de riesgo.

\section{INTRODUÇÃO}

A Síndrome da Morte Súbita do Lactente (SMSL) se caracteriza por morte repentina e inesperada de crianças menores de um ano de idade e que permanece sem alguma explicação, mesmo após investigação da história clínica, necrópsia completa e avaliação do local do óbito (GEMBLE A, et al., 2020; JENNAH M, et al., 2020; GOLDBERG N, et al., 2018). É considerada uma doença multifatorial que tem seu início mediado por condições de desenvolvimento, genéticas e ambientais (RAMIREZ LM, et al., 2018).

Apesar de a causa da SMSL ser desconhecida, ela também é chamada como a morte no berço enquanto o bebê dorme, e geralmente é encontrado morto após adormecer (PINHO et al., 2008). Visto que, o sono não é apenas um estado de repouso, há intensa atividade cerebral que está relacionada com o desenvolvimento psicomotor e cognitivo da criança, pois, nos primeiros meses de vida a criança passa mais da metade do tempo dormindo. Diante disso, alguns hábitos de sono apresentam-se como fatores de risco para a ocorrência da SMSL (OLIVEIRA AMDF, 2020). Dentre os fatores de risco mais importantes da SMSL, destacam-se os mecanismos de excitação do sono ruim, posição em decúbito ventral, infecções respiratórias, cardiopatias congênitas e exposição da criança ao cigarro e outras drogas (GOLDBERG N, et al., 2018).

A união entre os fatores de risco durante dois a quatro meses de idade pode tornar o período crítico para a criança causando uma disfunção da via respiratória parcial ou total, possui origem no sistema nervoso, imaturo, o qual controla a função cardiorespiratória e ocasiona consequente morte súbita, pelo "esquecimento" de respirar por parte do lactente (GOLDBERG N, et al., 2018). Nos países desenvolvidos como os EUA, a SMSL possui muita relevância nos índices de mortalidade dos lactentes, sendo que a faixa etária de maior risco encontra-se entre 2 a 5 meses de vida e a cada ano 4.500 mortes são classificadas como morte infantil súbita e inesperada (STEPHEN M, et al., 2015).

Entretanto, nos países em desenvolvimento como o Brasil, a prevalência da síndrome é pouco conhecida, o que tem impactado diretamente o esclarecimento da população sobre os fatores de risco e a promoção de campanhas direcionadas à prevenção. Uma análise retrospectiva concluiu que a SMSL não tem sido diagnosticada de forma correta, pois, no Brasil não havia nenhum caso registrado na região Sul, até o ano 2000. Além disso, a necropsia não é conduta obrigatória, o que dificulta a identificação da distribuição da síndrome, bem como retarda a implantação de medidas educativas e preventivas (GEIB LTC e NUNES ML, 2006; MAGDA LN et al., 2011).

Embora existam recomendações para diminuir o risco de incidência da SMSL, é necessário a ampla divulgação e conhecimento sobre as maneiras de prevenir tal doença, como a orientação de que os lactantes sejam colocados em decúbito dorsal para dormir e em superfícies firmes, tomar cuidado com a quantidade de objetos presentes no berço, como cobertores, almofadas, brinquedos e travesseiros pois pode levar a um superaquecimento da criança e também interferir na sua respiração (MAGED M e RIZZOLO D, 2018). 
Além disso, recomenda-se orientar aos pais que as crianças não devem ser expostas à fumaça do cigarro ou a mãe consumir drogas ou álcool e não tiver tido um acompanhamento de pré-natal adequado, visto que são fatores que aumentam o risco de SMSL (MAGED M e RIZZOLO D, 2018). É fundamental, também, a amamentação durante os 6 primeiros meses, a criança ter o cartão de vacina completo e atualizado sempre que necessário. Todas essas recomendações contribuem para a redução da incidência de SMSL durante o sono e ótimas condições para o desenvolvimento saudável de uma criança (GOLDBERG N, et al., 2018).

Diante do exposto, o objetivo deste estudo é realizar uma revisão bibliográfica com os estudos presentes na literatura, ampliando o conhecimento sobre a SMSL e seus fatores de risco, com a finalidade de aumento da prevenção contra a SMSL e consequente diminuição da mortalidade.

\section{REVISÃO BIBLIOGRÁFICA}

A SMSL é definida como morte repentina durante o primeiro ano de vida, sem causa específica após investigação minuciosa e sem nenhum indicativo de que o bebê apresenta qualquer risco de vida (GEMBLE A, et al., 2020). Cerca de $90 \%$ dos casos ocorrem nos primeiros seis meses de idade, sendo o pico apontado entre dois e quatro meses, com posterior declínio. Há considerável diferença entre grupos étnicos e raciais, sendo a SMSL duas a sete vezes mais comuns em nativos negros nos Estados Unidos (EUA) quando comparados a média nacional geral (RAMÍREZ LM, et al., 2018).

A incidência de SMSL varia com o passar dos anos e entre diferentes países. Casos de SMSL em 1000 nascidos vivos em 2004 na Holanda foram de 0.09, no Japão 0.19, no Canadá 0.24, na Inglaterra e vivos em 2015, País de Gales 0.32, nos EUA 0.55, na Argentina 0.47 e na Austrália 0.22 (HAUCK FR e TANABE KO, 2009). Em 1994, a American Academy of Pediatrics lançou uma campanha denominada Back to Sleep, incentivando os pais a colocarem seus filhos para dormir em decúbito dorsal. Desde então, a taxa de SMSL nos EUA decresceu mais de 50\%: de 1,3 por 1000 nascidos vivos em 1990 para 0,4 por 1000 nascidos. Apesar disso, nos últimos anos a taxa de SMSL permaneceu estagnada mesmo com os esforços de saúde pública voltados para o ambiente de sono do bebê e para os grupos de risco, fazendo com que a SMSL continue em $2^{\circ}$ lugar de morte pós-neonatal e em $4^{\circ}$ em mortalidade infantil nos EUA (GOLDBERG N, et al, 2018).

No Brasil não existem dados estatísticos suficientes que apontem a verdadeira incidência da SMSL, pois não existem estudos oficiais direcionados e os casos são subdiagnosticados, devido a diagnósticos errôneos que se assemelham com a síndrome, como, por exemplo, asfixia acidental, asfixia acidental posicional e outras causas indeterminadas (PINHO APS e NUNES ML, 2010).

\section{Principais causas e fatores de risco}

A SMSL tem causa mal definida e desconhecida, tendo fatores genéticos e ambientais envolvidos. Atualmente a teoria mais aceita é do "triplo risco", na qual fatores ambientais, infecciosos e genéticos agem em lactentes vulneráveis e em estágio crítico do desenvolvimento causam SMLS, mas que em crianças não vulneráveis não são capazes de gerar morte (BLASCO AM, et al., 2017).

Segundo Micthell EA, et al. (2020) uma das principais causas é a sufocação acidental e estrangulamento na cama. Objetos maciços no berço, como cobertores, travesseiros e protetores de berço são um importante fator de risco e por isso grande parte das crianças vítimas de SMSL são encontradas com a cabeça coberta por roupas de cama. Além disso, o compartilhamento de leito com os pais aumenta o risco de SMSL, principalmente em prematuros, baixo peso ao nascer, menor que 11 semanas de vida.

O risco do leito compartilhado também é maior quando os pais são fumantes ou usuários de álcool e drogas. A maioria dos lactentes são expostos a pelo menos 1 fator de risco durante o primeiro ano de vida (OLIVEIRA AMF, et al., 2019). Além disso, o tabagismo materno ao longo da gravidez e no ambiente familiar após o nascimento também contribuem para SMLS (SÁNCHEZ T, et al., 2020). Visto que o ambiente inseguro do sono está muito associado à SMSL, a posição prona para dormir está relacionada, principalmente aos 4 meses (BLASCO AM, et al., 2017).

As causas cardíacas são importantes na síndrome da morte súbita infantil. Durante a formação embrionária, diversas mudanças devem ocorrer no feto para que seu desenvolvimento seja ideal. Na rede 
vascular coronariana, deve haver involução dos sinusóides capilares no interior do miocárdio, aparecimento de botões endoteliais nos locais adequados e formação da aorta a partir da divisão do truncus arteriosus. Qualquer etapa que tenha alguma modificação pode levar a anomalias das coronárias e ser causa da SMSL. A anomalia da coronária direita é mais prevalente, contudo a anomalia da coronária esquerda é mais letal e está relacionada com $85 \%$ dos casos de morte súbita cardíaca relacionada artéria coronária com origem anómala na aorta (SILVA A, et al., 2017).

Além disso, causas cerebrais estão relacionadas com a síndrome da morte súbita infantil. Essas vítimas parecem portar alteração cerebral que prejudica a resposta crítica de excitação ao estresse hipóxico durante o sono. A aquaporina 4 (AQP4) é o principal canal de água da membrana do cérebro envolvido na regulação da homeostase da água. A distribuição de água no tecido neural costuma ser desregulada após lesão neural hipóxica. Isso seria relacionado à expressão de aquaporina 4 (AQP4), que está associado à homeostase da água, pois é o principal canal de água da membrana cerebral. O mecanismo da AQP4 na gênese da SMLS ainda precisa ser melhor esclarecido, mas até agora acham que o genótipo rs2075575 CT /TT representa um fator de risco. A explicação seria maior risco subclínico de convulsões e menor capacidade de eliminar líquido em caso de edema gerado por hipóxia (EIDAHL JML, et al., 2021).

Além desses fatores, o baixo nível socioeconômico e educacional também representa fator de risco para SMSL, devido a maior dificuldade de aplicar medidas preventivas, como a posição adequada para o lactente dormir. $O$ temor de que a posição supina aumenta possibilidade de broncoaspiração e gera mais despertares é maior em pais com menor nível educacional (RAMIREZ LM, et al., 2018).

\section{Prevenção e acesso à informação}

As recomendações acerca da prevenção foram desenvolvidas para reduzir o risco de SMSL, sendo necessárias algumas estratégias para eliminar os fatores de risco (RAMIREZ LM, et al., 2018). Os profissionais e equipes de saúde têm o papel de fazer as recomendações para a redução do risco de SMSL, orientar os pais e cuidadores desde o nascimento do bebê (SUBRAMONIAN A e FEATHERSTONE R, 2020).

A American Academy Pediatrics (AAP) publicou recentemente sobre a SMSL recomendações atualizadas para reduzir o número de mortes infantis. Essas recomendações foram baseadas em estudos caso-controle com crianças de até 1 ano de idade (GOLDBERG N, et al., 2018).

A posição de dormir é um grande fator de risco para SMSL, portanto, é importante a posição supina até 1 ano de idade como posição para dormir ou até que o bebê consiga rolar de uma posição para outra. Devem ser evitadas também a posição de lado e de bruços, pois essas posições estão relacionadas a limiares de excitação aumentados, hipercapnia, hipóxia e controle autonômico alterado (GOLDBERG N, et al., 2018). Recomenda-se que os bebês sejam colocados em decúbito ventral apenas para facilitar os marcos motores e evitar a plagiocefalia posicional (SUBRAMONIAN A e FEATHERSTONE R, 2020). O tabagismo também aumenta o risco de SMSL, visto que é prejudicial na gestação e após o nascimento. Portanto, deve ser orientado aos pais evitar fumar durante a gestação e após os primeiros 6 meses de vida do lactente (MAGED M e RIZZOLO D, 2018).

Além disso, não devem ser utilizados assentos de carros e carrinhos de bebê durante o sono, pois aumenta o risco de obstrução de vias aéreas e pode causar lesões, provocando morte não intencional. É recomendado que não seja usado acessórios de cama macios porque há risco de sufocamento, aprisionamento e estrangulamento. Diante disso, deve ser usada uma superfície firme e plana com um lençol para dormir. A recomendação é de que o quarto pode ser compartilhado sem compartilhar a cama, pois, em bebês menores de 4 meses está associado a altas taxas altas de SMSL e pode ser ocasionado pelo risco de sobreposição e risco de superaquecimento devido a colchões macios (GOLDBERG N, et al., 2018).

O mecanismo de proteção da chupeta não é claro, mas o seu uso favorece o controle da respiração e também a estabilidade cardiovascular, pois mantém a permeabilidade das vias aéreas (MAGED M e RIZZOLO D, 2018). Apesar do uso da chupeta ser adiado para não prejudicar a amamentação, está estabelecido que o uso dela está associado a menor risco de SMSL e não devem estar presas a cordões para evitar qualquer risco (GOLDBERG N, et al., 2018). Uma outra medida protetora para a SMSL é a amamentação exclusiva 
que faz com que os bebês acordem mais facilmente comparados com aqueles que tomam fórmula (RAMIREZ LM, et al., 2018). Além disso, é importante a amamentação que fornece citocinas e imunoglobulinas necessárias para proteção do bebê, principalmente quando a SMSL ocorre (MAGED M e RIZZOLO D, 2018).

Vale acrescer que as diretrizes atuais da Sociedade Europeia de Cardiologia incluem o tratamento da Apneia Obstrutiva do Sono (AOS) para prevenir a SMSL. Dessa forma, o tratamento e prevenção da AOS são fundamentais para a prevenção da morte súbita cardíaca do lactente (OTTAVIANI G e BUJA LM, 2020).

\section{Orientações aos pais e cuidadores e papel dos médicos}

O médico possui papel de colaborar na criação e direcionamento de estudos acerca da SMSL. Idealmente, diversas especialidades médicas são requisitadas para melhor condução e efetividade dos trabalhos científicos sobre o assunto. Dessa forma, é possível alcançar conhecimentos para a prevenção da morte súbita infantil que contemplem diversas áreas da medicina (BLASCO AM, et al., 2017). A partir disso, ocorre a disponibilização de informações que os médicos precisam conhecer para orientar seus pacientes acerca das melhores formas de prevenção da SMSL (MAGED M e RIZZOLO D, 2018).

Todos os profissionais da saúde possuem participação na abordagem preventiva dessa síndrome. Particularmente, o médico deve abordar aspectos do sono seguro com pais e cuidadores dos bebês, orientar sobre a cessação do tabagismo materno durante a gravidez, não tabagismo no ambiente domiciliar, responder dúvidas e corrigir equívocos que possam ocorrer durante as consultas (GOLDBERG N, et al., 2018). Além disso, é importante destacar que as populações de maior risco, ou seja, pais e cuidadores que não seguem as recomendações para um sono seguro infantil, precisam de uma atenção maior nos esforços da educação preventiva. Principalmente através da compreensão cultural que seus pacientes estão inseridos e os obstáculos que podem ser ultrapassados conjuntamente para proteção do lactente (MAGED M e RIZZOLO D, 2018).

A forma como o médico pode transmitir o conhecimento também é relevante. A recomendação do uso de aplicativos e vídeos relacionados à saúde infantil aos responsáveis pela criança é uma oportunidade para melhor fixação das informações preventivas. Uma vez que materiais impressos são menos atraentes, menos atualizados e muitas vezes são ignorados por pacientes. Usando modernos canais de comunicação e informação o acesso pode ser feito rapidamente e possui maior possibilidade de propagação a todos os pacientes de forma padronizada e atualizada (SONTAG JM, et al., 2020).

\section{CONSIDERAÇÕES FINAIS}

Dado o exposto, é importante reconhecer os fatores de risco para SMSL a fim de prevenir tal desfecho. A educação dos pais e cuidadores acerca da forma correta com que os lactentes devem ser colocados para dormir e como deve ser a segurança desse ambiente se mostrou eficaz em diminuir a mortalidade de lactentes por SMSL em diversos países, como os EUA. Dessa maneira, a prevenção tem se mostrado como a principal ferramenta de combate à SMSL devendo ser considerada o foco de todos os esforços para sua redução. Entretanto, é necessário ressaltar a limitação do estudo em encontrar, e portanto, retratar dados atualizados referentes à epidemiologia da SMSL no Brasil, por se tratar de um acontecimento subdiagnosticado e que, por vezes, acaba sendo atribuído erroneamente a outras etiologias.

\section{REFERÊNCIAS}

1. AHLERS-SCHMIDT, CR, et al. Infant Safe Sleep Promotion: Increasing Capacity of Child Protective Services Employees. Int. J. Environ. Res. Public Health, 2021; 18, 4227.

2. BARTICK M, et al. Trends in Breastfeeding Interventions, Skin-to-Skin Care, and Sudden Infant Death in the First 6 Days after Birth. The Journal of Pediatrics, 2020; 218: 11-15.

3. BLASCO AM, et al. Estudio y prevención de la muerte súbita infantil: Experiencia en la comunidad valenciana. CorSalud, 2017; 9(3): 137-142.

4. BERGER S, MACCALLI E. Strategies for the Prevention of Sudden Cardiac Death in Children and Adolescents. Pediatr Ann, 2015; 44(12): e292-e297. 
5. CARLIN RF, MOON RY. Risk Factors, Protective Factors, and Current Recommendations to Reduce Sudden Infant Death Syndrome: A Review. JAMA Pediatr, 2017; 171(2): 175-180.

6. CHAPUR VF, et at. Epidemiology of sudden unexpected death in infancy in Argentina: secular trend and spatial variation. Archive Argentinos de Pediatría, 2019; 117(3): 164-170.

7. EIDAHL JML, et al. Aquaporin 4 expression in the hippocampus in sudden infant death syndrome and sudden unexplained death in childhood. J Chem Neuroanat, 2021; 115: 101962.

8. GEMBLE A, et al. Knowledge assessment of sudden infant death syndrome risk factors in expectant mothers: $A$ prospective monocentric descriptive study. Archives de Pediatrie, 2020; 27(1): 33-38.

9. GEIB LTC, NUNES ML. Incidência da síndrome da morte súbita em coorte de lactentes. Jornal de Pediatria (Rio J.), 2006; 82(1): 21-26.

10. GOLDBERG N, et al. Sudden infant death syndrome: a review. Pediatric annals, 2018; 47(3): e118-e123.

11. GUTIÉRREZ C, et al. Muerte inesperada del lactante: Análisis de 591 casos. Archivos de Pediatría del Uruguay, Montevideo, 2017; 88(1): 12-18.

12. HAUCK FR, et al. Research Priorities in Sudden Unexpected Infant Death: An International Consensus. Pediatrics, 2017;140(2): e20163514.

13. HAUCK FR, TANABE KO. Beyond "Back to Sleep": Ways to Further Reduce the Risk of Sudden Infant Death Syndrome. Pediatr Ann, 2017; 46(8): 284-290.

14. IMDAD A, et al. Vitamin A supplementation for the prevention of morbidity and mortality in infants one to six months of age. Cochrane Database of Systematic Reviews, 2016; 9(9): CD007480.

15. JENNAH M, et al. Obstetricians' and Gynecologists' Communication Practices around Smoking Cessation in Pregnancy, Secondhand Smoke and Sudden Infant Death Syndrome (SIDS): A Survey. International Journal of Environmental Research and Public Health, 2020; 1-10.

16. MAGED M, RIZZOLO D. Preventing sudden infant death syndrome and other sleep-related infant deaths. Journal of the American Academy of PAs, 2018; 31(11): 25-30.

17. MAGDA ML, et al. Síndrome da morte súbita do lactente: aspectos clínicos de uma doença subdiagnosticada. Jornal de Pediatria (Rio J) 2001; 77(1): 29-34.

18. MITCHELL EA, et al. Geographic Variation in Sudden Unexpected Infant Death in the United States. The Journal of Pediatrics, 2020; 220: 49-55.

19. MONZÓ BA, et al. Estudio y prevención de la muerte súbita infantil: Experiencia en la comunidad valenciana. CorSalud, 2017; 9(3): 137-142.

20. MORENO MA. Reducing the Risk of Sudden Infant Death Syndrome. JAMA Pediatrics, 2017; 171(2): 204.

21. OLIVEIRA AMDF, et al. Risk and protective factors for sudden infant death syndrome. Revista Brasileira de Enfermagem, 2020; 73(2): e20190458.

22. OTTAVIANI G, BUJA LM. Pathology of unexpected sudden cardiac death: Obstructive sleep apnea is part of the challenge. Cardiovasc Pathol, 2020; 47: 107221.

23. PINHO APS, NUNES ML. Perfil epidemiológico e estratégias para o diagnóstico de SMSL em um país em desenvolvimento. Jornal de Pediatria (Rio J.), 2011; 87(2): 115-122.

24. PINHO APS, et al. Risk factors for sudden infant death syndrome in a developing country. Revista Saúde Pública. 2008; 42(3): 396-401.

25. PSAILA K, et al. Infant pacifiers for reduction in risk of sudden infant death syndrome. Cochrane Database Syst Rev. 2017; 4(4): CD011147.

26. RAMIREZ LM, et al. Síndrome de muerte súbita del lactante. Medicina Legal de Costa Rica, 2018; 35(1): 65-74.

27. SÁNCHEZ T, et al. Malos hábitos de sueño en lactantes: Factor de riesgo para síndrome de muerte súbita del lactante. Estudio piloto. Revista Chilena de Pediatria, 2020, 91(4): 529-535.

28. STEPHEN M, et al. Sudden Infant Death Syndrome. Am Fam Physician.2015; 91(11): 778-783.

29. SUBRAMONIAN A, FEATHERSTONE R. Interventions for the Prevention of Sudden Infant Death Syndrome and Sudden Unexplained Death in Infancy: A Review of Guidelines, 2020.

30. TIPENE-LEACH D, ABEL S. Innovation to prevent sudden infant death: the wahakura as an Indigenous vision for a safe sleep environment. Aust J Prim Health, 2019; 25(5): 406-409. 